\title{
Automatic registration of 2-D with 3-D imagery in urban environments *
}

\author{
Ioannis Stamos and Peter K. Allen, \\ Department of Computer Science, Columbia University, New York, NY 10027, \\ \{istamos, allen\}@cs.columbia.edu
}

\begin{abstract}
We are building a system that can automatically acquire $3 D$ range scans and $2 D$ images to build geometrically correct, texture mapped $3 D$ models of urban environments. This paper deals with the problem of automatically registering the $3 D$ range scans with images acquired at other times and with unknown camera calibration and location. The method involves the utilization of parallelism and orthogonality constraints that naturally exist in urban environments. We present results for building a texture mapped 3-D model of an urban building.
\end{abstract}

\section{Introduction}

This paper deals with the problem of automatic pose estimation \& calibration of a camera with respect to an acquired geometric model of an urban scene. The poseestimation is part of a larger system $[27,1]$ which constructs 3-D solid CAD models from unregistered range images. Our goal is to enhance the geometric model with photographic observations taken from a freely moving $2-D$ camera by automatically recovering the camera's position and orientation with respect to the model of the scene and by automatically calibrating the camera sensor. We propose a method which provides a solution for modeling buildings in urban environments. Most systems which recreate photo-realistic models of the environment by a combination of range and image sensing $[28,22,31,24]$ solve the range to image registration problem by fixing the relative position and orientation of the camera with respect to the range sensor (that is the two sensors are rigidly attached on the same platform). The major drawbacks of this approach are A) Lack of $2-D$ sensing flexibility, since the limitations of range sensor positioning (standoff distance, maximum distance) translate to constraints on the camera placement, and B) Static arrangement of sensors which means that the system can not dynamically adjust to the requirements of each particular scene (the camera sensor is precalibrated off-line). Also, the fixed approach can not handle the case of mapping of historical photographs on the models, something our method is able to accomplish.

\footnotetext{
*Supported in part by an ONR/DARPA MURI award ONR No0014-95-1-0601 and NSF grants CDA-96-25374 and EIA-9729844.
}

This paper provides a solution to the automated pose determination of a camera with respect to a range sensor without placing artificial objects in the scene and without a static arrangement of the range-camera system. This is done by solving the problem of automatically matching $3-\mathrm{D} \& 2-\mathrm{D}$ features from the range and image data sets. Our approach involves the utilization of parallelism and orthogonality constraints that naturally exist in urban environments in order to extract $3-\mathrm{D}$ rectangular structures from the range data and 2-D rectangular structures from the $2-\mathrm{D}$ images.

The problems of pose estimation and camera calibration are of fundamental importance in computer vision and robotics research since their solution is required or coupled with stereo matching, structure from motion, robot localization, object tracking and object recognition algorithms. There are numerous approaches for the solution of pose estimation problem from point correspondences $[10,8,20,7,23]$, or from line correspondences $[17,12,6]$. Work in automated matching of $3-D$ with 2-D features include $[13,19,21,5,29,14,11,16]$ whereas in [30] the automated matching is possible when artificial markers are placed in the scene.

\section{Problem Formulation}

Formally, our input consists of the pair $(D(S), \mathbf{I}(S))$ of a scene's $S$ range scan $D$ and set of images $I$. We assume that both the camera \& range sensors view the same part of the real scene, so that the $3-\mathrm{D}$ and $2-\mathrm{D}$ views have significant overlap (figure 1). The locations of the cameras which produce the images I is unknown and must be automatically recovered. Thus the output is the pose $P_{i}=\left\{R_{i}, \mathbf{T}_{\mathbf{i}} \mid \mathbf{P p}_{\mathbf{i}}, f_{i}\right\}$ which describes (a) the transformation (rotation $R_{i} \&$ translation $\mathbf{T}_{\mathbf{i}}$ ) from the range-sensor to each camera-sensor's coordinate system and (b) the mapping (internal camera parameters) from the 3-D camera frames of reference to the 2-D image frames of reference (we optimize wrt the principal point $\mathbf{P} \mathbf{p}_{\mathbf{i}}$ and focal length $f$ and we assume no distortion).

The pose estimation involves the following stages.

A) Extraction of two feature sets $F_{3 D}$ and $F_{2 D}(3-$ $D \& 2-D$ linear segments from the range and image data-sets) [27]. B) Grouping of the 3-D and 2-D feature sets into clusters of parallel $3-\mathrm{D}$ lines $L_{3 D}$ and 
converging 2-D lines ${ }^{1} L_{2 D}$ (global properties) [section 3]. C) Computation of an initial pose estimate $P_{0}=\{R, \mathbf{0} \mid \mathbf{P} \mathbf{p}, f\}$ (rotation and internal camera parameters) by utilizing the directions defined by the sets $L_{3 D}$ and $L_{2 D}$ [section 4]. D) Grouping of the 3-D and 2-D line segments into higher level structures of 3-D and 2-D rectangles $R_{3 D}$ and $R_{2 D}$ (local properties) and extraction of 3-D and 2-D graphs $G_{3 D}$ and $G_{2 D}$ of rectangles (repetitive pattern of scene and image rectangles) [section 5]. E) Automatic selection of a matched set of rectangular features $C^{\circ}$ and computation of a pose $P^{o}=\mathcal{A}\left(C^{0} \mid P_{0}\right)$ by running a pose estimator algorithm $\mathcal{A}$ (computation of a coarse pose estimate). Refinement $P^{R}=R\left(P^{o}, L_{3 D}, L_{2 D}\right)$ of the estimated pose $P^{o}$ by using all available information computed so far (computation of a fine pose estimate) [section 6].

The following sections describe all steps in more detail.

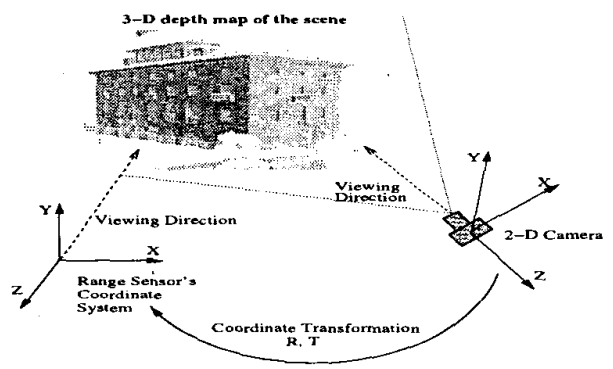

Figure 1: The pose estimation problem. The 3-D model of the scene is represented in the coordinate system of the range sensor. The image taken from the 2-D camera needs to be registered with the 3-D model.

\section{Vanishing Point Extraction \& Clus- tering 3-D lines}

Previously we have developed robust methods for generating 3-D and 2-D line-sets from 3-D and 2-D imagery [27]. In this paper, matched 2-D \& 3-D clusters of lines are used for recovering rotation and for camera internal self-calibration. In the 2-D domain the extraction of vanishing points provides a natural clustering of lines into sets which correspond to parallel 3-D lines whereas in the 3-D domain the clustering into sets of parallel 3-D lines is direct.

The most characteristic property of perspective projection is the fact that a set of parallel $3-\mathrm{D}$ lines is mapped to a set of $2-D$ lines which intersect at a common point on the image plane. This point of intersection can be a point at infinity when the corresponding $3-\mathrm{D}$ direction is parallel to the image plane. In order to handle all possible points of intersection (even points at infinity) we need to represent $2-D$ points and $2-D$

\footnotetext{
${ }^{1}$ Those lines define vanishing points on the image space.
}

lines as antipodal-points in the Gaussian sphere Then, the intersection of two 2-D lines $\mathbf{l}_{\mathbf{1 2}}$ (represented with the two antipodal points $\pm \mathbf{N}_{12}$ on the sphere) and $\mathbf{l}_{\mathbf{1 2}}^{\prime}$ $\left( \pm N_{12}^{\prime}\right)$ is the $2-D$ point $\mathbf{v}$ which is mapped to the antipodal points $\pm \mathbf{N}_{12} \times \mathbf{N}_{12}^{\prime}$ on the Gaussian sphere and can be represented by a pair $(\phi, \theta)$, where $0 \leq \phi \leq 2 \pi$ and $0 \leq \theta \leq \pi / 2[25]$.

There are many methods for the automatic computation of the major image vanishing points (see $[2,4,18]$ ). Our approach involves the computation of all pairwise intersections between the extracted image lines and the creation of a 2-D histogram of those intersections. The histogram is defined over the 2-D domain of the discretized surface of the Gaussian sphere. Then a search for the peaks of the histogram is performed. Each peak corresponds to directions towards which a large number of 2-D lines converge.

The end result is a set of major vanishing points $\mathbf{V P}=\left\{v_{1}, \ldots, v_{n}\right\}$, where $V P_{i}=\left(\phi_{i}, \theta_{i}\right)$ Each vanishing point is supported by a set of 2-D lines and the desired clustering $\mathrm{L}_{2 \mathrm{D}}=\left\{L_{2 D_{1}}, \ldots, L_{2 D_{n}}\right\}$ has been accomplished. If the number of major vanishing points $N_{v p s}$ is known a-priori (in urban environments this number is almost always three) then we can select the $N_{v p s}$ largest clusters from the set $\mathbf{L}_{\mathbf{2 D}}$ as our result and so $\mathbf{L}_{\mathbf{2 D}}=\left\{L_{2 D_{1}}, \ldots, L_{2 D_{N_{v p s}}}\right\}$ and $\mathbf{V P}=\left\{v_{1}, \ldots, v_{N_{v p s}}\right\}$. Extracting the number $N_{v p s}$ is an easy task (it is equivelant to identifying the major modes of the $1-D$ histogram of directions of 2-D lines on the plane [18] ).

The clustering of the extracted 3-D lines into sets of parallel lines is an easier task than the extraction of vanishing points. We are using a classic unsupervised nearest neighbor clustering algorithm [15]. The $N_{v p s}$ larger clusters of $3-\mathrm{D}$ lines provide the desired grouping of $3-\mathrm{D}$ lines into clusters of parallel lines $\mathbf{L}_{3 \mathrm{D}}=\left\{L_{3 D_{1}}, \ldots, L_{3 D_{N_{v p s}}}\right\}$ along with the average $3-\mathrm{D}$ direction of each cluster $\mathbf{U}_{3 \mathbf{D}}=\left\{V_{3 D_{1}}, \ldots, V_{3 D_{N_{v p}}}\right\}$.

\section{Initial pose estimation}

The rotation computation is based on the fact that the relative orientation between two $3-\mathrm{D}$ coordinate systems $O$ and $O^{\prime}$ can be computed if two matching directions between the two systems are known. In this case there is a closed-form solution for the rotation [9] and we can write $R=R\left(\mathbf{n}_{1}, \mathbf{n}_{1}^{\prime} \mid \mathbf{n}_{2}, \mathbf{n}_{2}^{\prime}\right)$, where $\mathbf{n}_{\mathbf{i}}$ and $\mathbf{n}_{\mathbf{i}}^{\prime}$ are corresponding orientations expressed in the coordinate systems $O$ and $O^{\prime}$. In our case, the direction of the $3-\mathrm{D}$ lines which produce the vanishing point $v_{i}$ is the unit vector $\mathbf{n}_{\mathbf{i}}=\left(v_{i}-C O P\right) /\left\|\left(v_{i}-C O P\right)\right\|(C O P$ is the center of projection of the camera), expressed in the coordinate system of the camera sensor (section 3 ). This direction can be matched with a scene direction $\mathbf{n}_{\mathbf{i}}^{\prime}$ which is expressed in the coordinate system of the range sensor and which has been provided by the 3-D clustering module (section 3). So, the rotation computation is reduced to the problem of finding two pairs of matching 
3-D directions \& 2-D vanishing points

$$
\left(\mathbf{n}_{\mathbf{i}}^{\prime}, \mathbf{n}_{\mathbf{i}}\right) \epsilon \mathbf{U}_{\mathbf{3 D}} \times \mathbf{V P} \text {. }
$$

The camera center of projection (principal point and focal length) can be computed by three such pairs of directions $^{2}$ (see $\left.[4,3]\right)$.

\section{Extracting 3-D \& 2-D rectangles}

Calculating the translation requires the exact matching of local 3-D and 2-D features and global properties alone are not enough. Since $3-\mathrm{D}$ points are hard to localize in the 3-D data set and since we have already developed a method for the reliable and accurate extraction of 3-D lines [27] we will match 2-D with 3-D linear features. In order to reduce the search-space of possible matches we move up in the feature hierarchy and group the 3-D and 2-D lines into graphs of rectangular \& quadrangular structures.

The geometry of the projection of a $3-\mathrm{D}$ rectangle on a 2-D image quadrangle is shown in figure 2. 3-D rectangles which are formed by pairs of lines of directions $\left(V_{\text {ver }}, V_{\text {hor }}\right)$ have corresponding 2-D quadrangles which are formed by pairs of 2-D lines which converge to the vanishing points $\left(v_{v e r}, v_{h o r}\right)$. That means that in order to extract corresponding $3-\mathrm{D}$ rectangles \& 2-D quadrangles we need to utilize the extracted clusters of 3-D \& 2-D lines.

For the following discussion we will call one of the two scene directions vertical $\left(V_{v e r}\right)$ and the other one horizontal $\left(V_{h o r}\right)$. We assume that the vertical direction is oriented from the bottom to the top of the scene whereas the horizontal from left to right. Analogously we call $v_{v e r}$ and $v_{h o r}$ the vanishing points which correspond to the directions $V_{v e r}$ and $V_{\text {hor }}$.

We can formulate the $3-D$ and $2-D$ rectangle extraction problem as follows: The input is two pairs of $3-$ D directions $V_{v e r}, V_{h o r} \epsilon \mathbf{U}_{\mathbf{3 D}}$ and 2-D vanishing points $v_{v e r}, v_{h o r} \epsilon \mathbf{V P}$ along with the $3-\mathrm{D} L_{3 D_{0}}, L_{3 D_{1}} \in \mathbf{L}_{3 \mathbf{D}}$ and 2-D $L_{2 D_{0}}, L_{2 D_{1}} \in \mathbf{L}_{\mathbf{2}} \mathbf{D}$ (section 3 ) clusters that support them. The output is a set of $3-D$ rectangles $\&$ 2-D quadrangles $R_{3 D}$ and $R_{2 D}$ and two corresponding graphs $G_{3 D}$ and $G_{2 D}$ describing the spatial relationship among structures in $R_{3 D}$ and $R_{2 D}$ respectively.

Following, this notation a $3-D$ rectangle is a planar 3-D structure whose sides can be tagged as $l_{u p}$ or $l_{\text {down }}$ if are parallel to the $V_{h o r}$ direction and as $l_{\text {left }}$ or $l_{\text {right }}$ if are parallel to the $V_{v e r}$ direction (figure 2). Also we can define three relationships between rectangles which lie on the same scene plane: right of, top of and in or out of. The exact same representation can be used for the 2-D quadrangles. In order to use the same notation and define spatial relationships between $2-\mathrm{D}$ quadrangles we

\footnotetext{
${ }^{2}$ The camera calibration is performed before the computation of the rotation, since the rotation computation assumes a calibrated camera.
}

need to transform them to $2-\mathrm{D}$ rectangles. This can be done if we rotate the two vanishing points $v_{v e r}$ and $v_{\text {hor }}$ (and similarly transform all 2-D lines which they support them) such that they are parallel to the image plane.

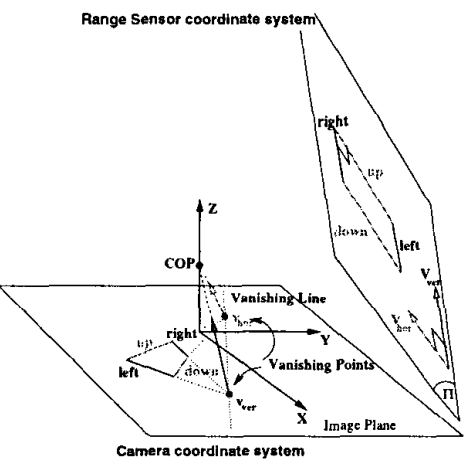

Figure 2: 3-D rectangle formed by lines parallel to the scene directions $V_{v e r}$ and $V_{h o r}$ and its corresponding 2$\mathrm{D}$ quadrangle formed by $2-\mathrm{D}$ lines which meet at the image vanishing points $v_{v e r}$ and $v_{\text {hor }}$.

The 3-D rectangle and 2-D quadrangle algorithms are almost identical. They differ in the following manner: 2-D case: Quadrangles (instead of rectangles) need to be extracted (see figure 2). However, with vanishing points already computed it is possible to undo the perspective effect and map quadrangles to rectangles. 3-D case: A check for coplanarity of the linear segments that form the borders of the rectangle is required.

We present an algorithm that can be applied in both 2-D and 3-D cases. The vertical and horizontal lines are directed according to the $V_{v e r}$ and $V_{h o r}$ orientations (figure 2). Thus each line can be represented as a pair of points $\left(P_{\text {start }}, P_{\text {end }}\right)$. The major steps of the algorithm are: A) Traverse all vertical lines $\left(P V_{\text {start }}, P V_{\text {end }}\right)$ and record their closest horizontal lines $\left(P H_{\text {start }_{i}}, P H_{\text {end }}\right)$. The distance between a vertical and a horizontal line is defined as the distance between their closest endpoints. Horizontal lines whose distance is greater than max (user supplied threshold) are not considered as candidates for closeness. B) Traverse the horizontal lines and check for patterns of four, three or two sided rectangles by utilizing the spatial relationships extracted in the previous step ${ }^{3}$. C) Compute the graphs that describe the spatial relationships among rectangles. More details can be found in [26].

Concluding, we have formulated and solved the problem of extracting $3-\mathrm{D} \& 2-\mathrm{D}$ rectangles from pairs of $3-$ D directions $\left(V_{v e r}, V_{h o r}\right) \epsilon \mathbf{U}_{3 \mathbf{D}}$ and their matching pairs

\footnotetext{
${ }^{3}$ Normally, our input consists of lines which do not define complete four-sided rectangles. That is why we allow the representation and extraction of incomplete rectangles.
} 
of 2-D vanishing points $\left(v_{v e r t}, v_{h o r}\right) \epsilon \mathbf{V P}$ (section 3). The output of this module is pairs of sets of $3-\mathrm{D}$ and $2-\mathrm{D}$ rectangles $\left(R_{3 D_{I}}, R_{2 D_{I}}\right)$. In section 6 we will describe how we utilize the extracted sets of rectangles for the computation of a coarse pose estimate.

\section{Pose Estimation}

The last part of the pose computation module is the calculation of the camera translation with respect to the range sensor by matching local 3-D \& 2-D features between the range and image data sets.

In section $43-\mathrm{D}$ scene directions are matched with 2-D image vanishing points in order to solve for the camera rotation. If we have $N$ such matches $\left(\mathbf{n}_{\mathbf{i}}^{\prime}, \mathbf{n}_{\mathbf{i}}\right)$ of scene and image directions then there are $M=\left(\begin{array}{c}N \\ 2\end{array}\right)$ pairs of the form $\left(\left(\mathbf{n}_{\mathbf{i}}^{\prime}, \mathbf{n}_{\mathbf{i}}\right),\left(\mathbf{n}_{\mathbf{j}}^{\prime}, \mathbf{n}_{\mathbf{j}}\right)\right)$. In section 5 we described a method to compute $3-D$ \& 2-D rectangles $\left(R_{3 D_{k}}, R_{2 D_{k}}\right)$ from clusters of $3-\mathrm{D}$ and $2-\mathrm{D}$ lines, and pairs of the above pairs of matched scene and image directions. Since we have $M$ such pairs, we can compute $M$ pairs of sets of 3-D \& 2-D rectangles $\left(R_{3 D_{k}}, R_{2 D_{k}}\right)$ and the set $\mathcal{S}=\mathcal{P}\left(R_{3 D_{1}} \times R_{2 D_{1}}\right) \cup \ldots \cup \mathcal{P}\left(R_{3 D_{M}} \times\right.$ $\left.R_{2 D_{M}}\right)^{4}$ describes the space of every possible matching configuration between $3-\mathrm{D}$ and $2-\mathrm{D}$ rectangles.

Matching between a set of 3-D and 2-D rectangles will provide us a coarse pose estimate. Exploring every possible combination of matches is an intractable problem since we need to consider an exponentially large number of possibilities. In order to solve the problem we follow the RANSAC framework introduced in [10]. Instead of considering all possible matches we are randomly sampling the search space $\left(R_{3 D_{1}} \times R_{2 D_{1}}\right) \cup\left(R_{3 D_{2}} \times R_{2 D_{2}}\right) \cup \ldots \cup\left(R_{3 D_{M}} \times R_{2 D_{M}}\right)$ of 3-D and 2-D rectangular structures. Each sample $C_{\text {ran }}$ consists of a fixed number $n_{\text {ran }}$ of pairs of $3-\mathrm{D}$ and 2$\mathrm{D}$ rectangles, where $n_{r a n}$ is the minimum number of matches that can produce a reliable pose-estimate. Every sample $C_{\text {ran }}$ produces a pose estimate which is being verified and a matching score $Q_{\text {match }}$ is computed, and we select as correct the match which produces the maximum. Our algorithm sets the score $Q_{\text {match }}$ to equal the number of 3-D rectangles which map (when projected to the image) to an extracted 2-D quadrangle (larger is better). What remains to be defined is how do we decide when two 2-D rectangles are close with respect to each other ${ }^{5}$. This decision is based on an adaptive threshold which depends on the relative size of pairs of rectangles. Finally, the pose estimation algorithm $\mathcal{A}$ from a set of matched 3-D and 2-D lines (we can view each rectangle as a set of four lines) is described in detail in [17]. In the implementation of the RANSAC procedure the pose estimator $\mathcal{A}$ optimizes only with respect to the

\footnotetext{
${ }^{4} \mathcal{P}(A)$ is the powerset of a set $\mathrm{A}$.

${ }^{5}$ Note that 2-D quadrangles are transformed to $2-\mathrm{D}$ rectangles when we extract the vanishing points which produce them.
}

translation since the rotation is already known to us.

If we want to ensure with probability $\mathrm{Pr}$ that at least one of our random selections corresponds to a valid match then the maximum number of steps is $N_{\max }=\log (1-P r) / \log (1-b)$ where $b$ is the probability of randomly selecting a sample of $n_{\text {ran }}$ correct matches [10]. If we assume that in our scene there are $K$ pairs of $3-\mathrm{D}$ and $2-\mathrm{D}$ rectangles that can be correctly matched then $b=(K / L)^{n_{\text {ran }}}$ and $L=$ $\left|\left(R_{3 D_{1}} \times R_{2 D_{1}}\right) \cup\left(R_{3 D_{2}} \times R_{2 D_{2}}\right) \cup \ldots \cup\left(R_{3 D_{M}} \times R_{2 D_{M}}\right)\right|$ is the number of all possible pairs of $3-D$ and $2-D$ rectangles. $K$ is unknown to us we set it equal to $1 / 3 L$ for our experiments. Note that the lower the probability of correct matches $b$ the larger the number of required steps $N_{\max }$.

The coarse estimate computed using the RANSAC method is very important because it provides an initial solution which can be subsequently refined and lead to a final pose estimate. The refinement involves the projection of all 3-D lines of the extracted clusters $\mathbf{L}_{\mathbf{3 D}}$ on the 2-D image assuming the coarse pose estimate $P^{o}$ and so a set of projected $3-\mathrm{D}$ lines $\mathcal{P}\left(\mathbf{L}_{\mathbf{3 D}}\right)$ is formed. Each individual projected cluster is compared with the groups of extracted $2-\mathrm{D}$ lines $\mathbf{L}_{\mathbf{2 D}}$ and new line matches among the 3-D and 2-D data sets are verified. The increased number of line matches results in better pose estimation.

\section{Results}

The recovered 3-D model of the urban building is shown in figure $3 \mathrm{a}$. In figures $3 \mathrm{~b}$ and $3 \mathrm{c}$ the clustering of the automatically extracted $3-\mathrm{D}$ lines is presented along with the computed $3-\mathrm{D}$ rectangles (the clustering is shown for two different views of the model). The three major vanishing points and clusters of 2-D lines are shown in figures $4 \mathrm{a}$ and $4 \mathrm{~b}$. The automatically computed principal point of the cameras is also shown; it is the point of intersection of vanishing point directions on the image. The next set of figures $(4 c, 4 d)$ displays the results of $2-D$ rectangle extraction and the outcome of the coarse pose estimation algorithm. The extracted $2-\mathrm{D}$ rectangles (black) are shown overlaid with the projection (white) of those $3-D$ rectangles which produce the maximum matching score $Q_{\text {match }}\left(Q_{\text {match }}\right.$ is 9 for the first view and 8 for the second view). The final pose (section 6) is visually verified in figures $4 \mathrm{e}$ and 4f where the extracted 3-D lines shown in figures 3c and $3 \mathrm{~d}$ respectively are projected on the 2 -D images assuming the final pose (shown in white). The extracted 2-D lines are shown in black. As you can see the projected $3-\mathrm{D}$ lines are very well aligned with the 2-D data-sets, which means that both the registration and the feature extraction algorithms produce accurate results. The number of samples the RANSAC algorithm tried was 8457 ( 6 seconds on an Onyx2) for the first view and 223831 ( 2 minutes and 29 seconds) for 

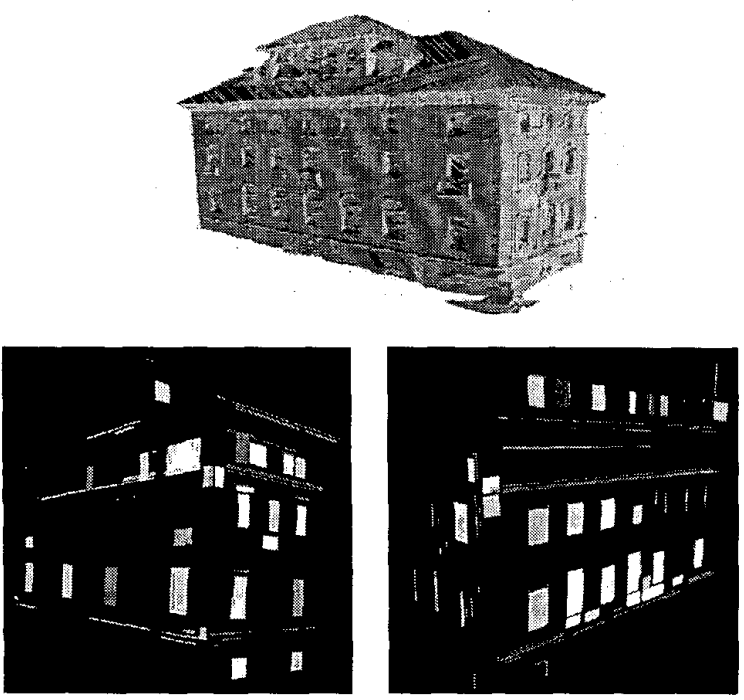

Figure 3: a) 3-D model of the scene from [27]. b,c) Clusters of 3-D lines (gray-level encodes different directions) and extracted 3-D rectangles (rectangles are rendered as solids of different color for clarity).

the second view. Finally, the two images in figure 5 present the texture-mapped 3-D models using the computed calibration parameters and pose estimate on the two views of the model. The texture map, also visually verifies the accuracy of our method. The final pose estimates are $\mathbf{T}=(3.71,-2.93,12.29)^{T}$ (in meters), $R=\left\{175.65^{\circ},(0.017,0.99,0.01)^{T}\right.$ (angle-axis representation) for the first view and $\mathbf{T}=(1.35,-2.5,10.10)^{T}$, $R=\left\{178.86^{\circ},(0.0,0.99,0.01)^{T}\right.$ for the second.

\section{Summary}

We have developed a method to accurately register a range with an image data set in urban environments. We are exploiting the parallelism and orthogonality constraints that naturally exist in such environments in order to match extracted sets of rectangular structures. The use of a RANSAC technique for the computation of an optimal match between the data-sets is feasible due to the reduction of the search space from the set of $3-\mathrm{D}$ and 2-D lines to the set of 3-D and 2-D rectangles. One problem with RANSAC is the computation of maximum number of steps that must be performed. The method is completely automatic, except of the assumption that we know the match between the $N_{v p s}$ (in our case 3) scene and vanishing point directions.

\section{References}

[1] P. K. Allen, I. Stamos, A. Gueorguiev, E. Gold, and P. Blaer. Avenue: Automated site modeling in urban environments. In 3rd Int. Conf. on Dig. Im. and Mod., 2001.

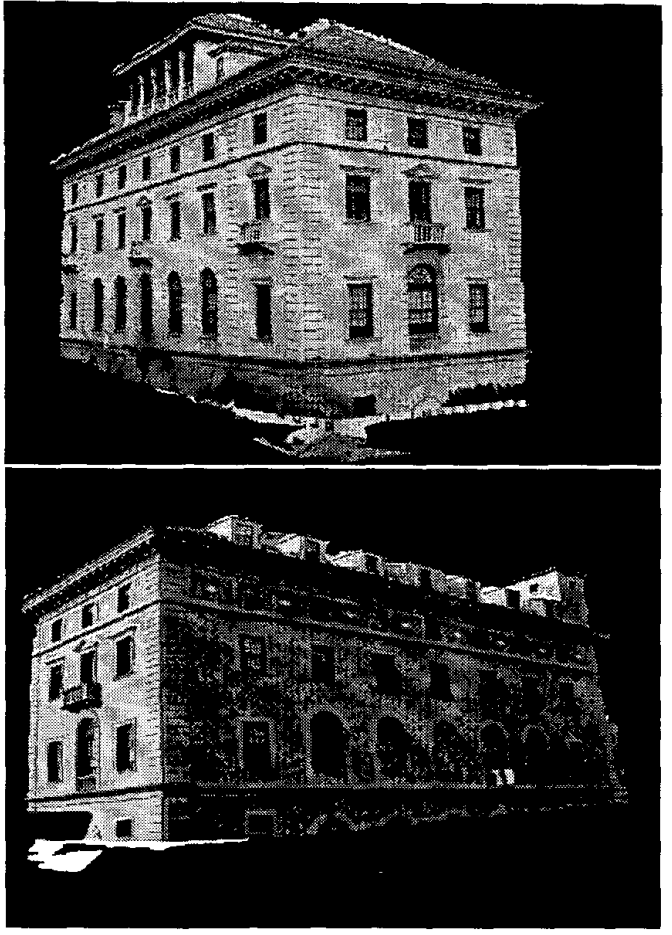

Figure 5: Images texture-mapped on 3-D model assuming final pose.

[2] M. E. Antone and S. Teller. Automatic recovery of relative camera rotations for urban scenes. In CVPR, pages 282-289, Hilton Head, NC, July 2000.

[3] S. C. Becker. Vision-assisted modeling from modelbased video representations. $\mathrm{PhD}$ thesis, MIT, Feb. 1997.

[4] B. Caprile and V. Torre. Using vanishing points for camera calibration. ICC $V, 4: 127-140,1990$.

[5] T. Cass. Polynomial-time geometric matching for object recognition. IJCV , 21(1-2):37-61, 1997.

[6] S. Christy and R. Horaud. Iterative pose computation from line correspondences. CVIU, 73(1):137-144, January 1999.

[7] D. F. DeMenthon and L. S. Davis. Model-based object pose in 25 lines of code. IJCV, 15:123-141, June 1995.

[8] M. Dhome, M. Richetin, J.-T. Lapresté, and G. Rives. Determination of the attitude of 3-D objects from a single perspective view. PAMI, 11(12):1265-1278, 1989.

[9] O. Faugeras. Three-Dimensional Computer Vision. The MIT Press, 1996.

[10] M. A. Fischler and R. C. Bolles. Random sample consensus: A paradigm for model fitting with applications to image analysis and automated cartography. Graphics and Image Processing, 24(6):381-395, June 1981.

[11] G. Hausler and D. Ritter. Feature-based object recognition and localization in 3D-Space, using a single video image. CVIU, 73(1):64-81, 1999. 

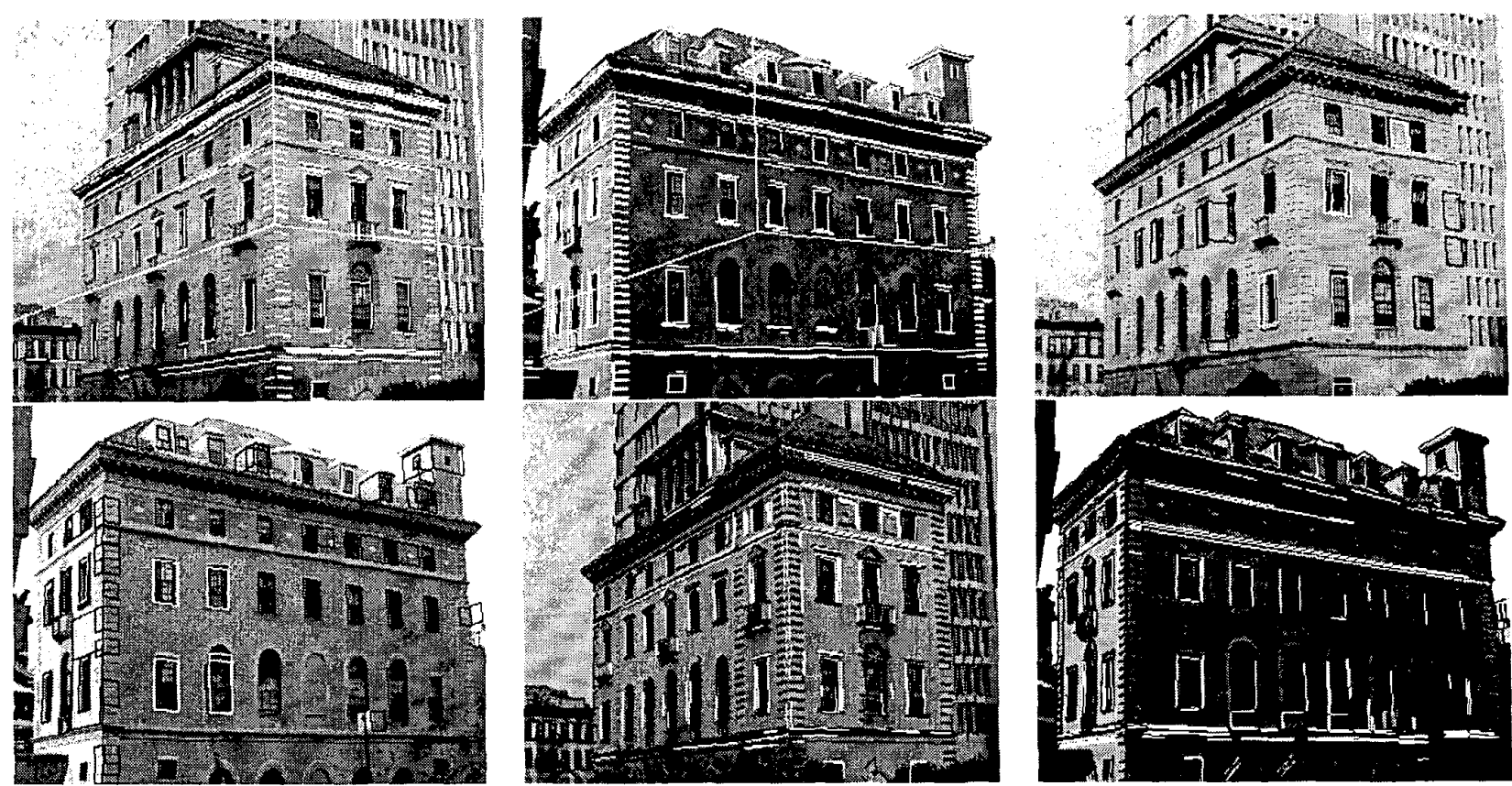

Figure 4: Results. a,b) 2-D images, clusters of 2-D lines and three major vanishing point directions (different vanishing points should appear in different color, something very hard to show in black and white printing). c,d) Extracted 2-D quadrangles (shown in black) and $Q_{\text {match }}$ matched 3-D rectangles projected on images after coarse pose estimation (shown in white). e,f) Projected 3-D lines on the images after final pose estimation (shown in white). The extracted 2-D lines are shown in black. Color images can be found in http://www.cs.columbia.edu/robotics.

[12] R. Horaud, F. Dornaika, B. Lamiroy, and S. Christy Object pose: The link between weak perspective, paraperspective, and full perspective. IJCV, 22(2), 1997.

[13] D. Huttenlocher and S. Ullman. Recognizing solid objects by alignment with an image. IJCV, 5(7):195-212, 1990.

[14] D. W. Jacobs. Matching 3-D models to 2-D images. IJCV , 21(1-2):123-153, 1997.

[15] A. Jain and R. Dubes. Algorithms for Clustering Data. Prentice-Hall, 1988.

[16] F. Jurie. Solution of the simultaneous pose and correspondence problem using gaussian error model. CVIU, 73(3):357-373, March 1999.

[17] R. Kumar and A. R. Hanson. Robust methods for estimating pose and a sensitivity analysis. CVGIP, 60(3):313-342, Nov. 1994.

[18] D. Liebowitz and A. Zisserman. Metric rectification for persepective images of planes. In $C V P R$, pages $482-$ 488, Santa Barbara, CA, 1998.

[19] D. Lowe. Robust model-based motion tracking through the integration of search and estimation. IJCV, $8(2): 113-122,1992$.

[20] D. Oberkampf, D. DeMenthon, and L. Davis. Iterative pose estimation using coplanar feature points. CVGIP, 63(3), May 1996.

[21] C. Olson. Time and space efficient pose clustering. In CVPR, pages 251-258, Seattle, WA, 1994.
[22] K. Pulli, H. Abi-Rached, T. Duchamp, L. G. Shapiro, and W. Stuetzle. Acquisition and visualization of colored 3-D objects. In ICPR, Australia, 1998.

[23] L. Quan and Z. Lan. Linear N-point camera pose determination. PAMI, 21(7), July 1999.

[24] V. Sequiera, K. Ng, E. Wolfart, J. Concalves, and D. Hogg. Automated reconstruction of 3D models from real environments. ISPRS Journal of Photogrammetry E. Remote Sensing, 54:1-22, 1999.

[25] C. Springer. Geometry and analysis of projective spaces. Freeman, 1964.

[26] I. Stamos. Geometry and Texture Recovery of Scenes of Large Scale. PhD thesis, Columbia University, 2001.

[27] I. Stamos and P. K. Allen. 3-D model construction using range and image data. In CVPR, Hilton Head, SC, July 2000.

[28] Visual Information Technology Group, Canada, 2000. http://www.vit.iit.nrc.ca/VIT.html.

[29] W. Wells. Statistical approaches to feature-based object recognition. IJCV, 21(1-2):63-98, 1997.

[30] Y. Yu. Modeling and Editing Real Scenes with ImageBased Techniques. PhD thesis, UC Berkeley, 2000.

[31] H. Zhao and R. Shibasaki. A system for reconstructing urban 3D objects using ground-based range and CCD sensors. In Urban Multi-Media/3D Mapping workshop, Inst. of Industr. Sc., The Univ. of Tokyo, 1999. 